\title{
Detection of 14 high-risk human papillomaviruses using digital LAMP assays on a self-digitization chip
}

Jiasi Wang ${ }^{\&} \uparrow \S$, Jeannette P. Staheli\& $\ddagger$, Andrew Wu $\ddagger$, Jason E. Kreutz $\dagger$, Qiongzheng Hu $\dagger$, Jingang Wang $\dagger$ , Thomas Schneider $\dagger$, Bryant S. Fujimoto $\dagger$, Yuling Qin $\dagger$, Gloria S. Yen $\dagger$, Bob Weng $\dagger$, Kara Shibley†, Halia Haynes $\dagger$, Rachel L. Winer\#, Qinghua Feng // , Daniel T. Chiu*†.

$\dagger$ Department of Chemistry, University of Washington, Seattle, Washington 98195, United States $\ddagger$ Center for Global Infectious Disease Research, Seattle Children's Research Institute, Seattle, WA, USA

\# Department of Epidemiology, University of Washington, Seattle, WA, 98195, USA

// FIDALAB, Seattle, WA, USA

\section{Materials and Methods}

Table S1 Final LAMP primer sequences

\begin{tabular}{lllc}
\hline HPV type & Primer & Sequence & Target \\
\hline HPV 16 & HPV16 F3 & AAGGCTCTGGGTCTACTG & L1 \\
& HPV16 B3 & CCATGTCGTAGGTACTCCT & \\
& HPV16 FIP & GGCCCTGTGCTCGTTGTAATTTACACCTAGTGGTTCTATGGT & \\
& HPV16 BIP & TGGCATTTGTTGGGGTAACCATTTTATGGCAGCACATAATGACAT & \\
& HPV16 LF & TGAATATTTGGGCATCAGAGGT & \\
& HPV16 LB & ACTGTTGTTGATACTACACGCA & \\
\hline HPV 18 & HPV18 F3 & CGCGTCCTTTATCACAGG & \\
& HPV18 B3 & TGGAATCCCCATAAGGATC & \\
& HPV18 FIP & GGCACCATATCCAGTATCTACCATAATTGCCCCCCTTTAGAACT & \\
& HPV18 BIP & AGGAT & \\
& HPV18 LF & TCACCATCTTCCAAAACTG & \\
& HPV18 LB & ATTGGATATTTGTCAGTCT & \\
\hline
\end{tabular}




\begin{tabular}{|c|c|c|c|}
\hline \multirow{12}{*}{ HPV 33} & HPV33_12 & & \\
\hline & F3 & AGAAACTGCACTGTGACG & E6/E7 \\
\hline & HPV33_12 & & \\
\hline & B3 & GTAGTTGCTGTATGGTTCGT & \\
\hline & HPV33_12 & & \\
\hline & FIP & CGGTCCAAGCCTTCATCCTCTTTTTAATCCTGAACCAACTGACCTA & \\
\hline & HPV33_12 & & \\
\hline & BIP & ACAAGCACAACCAGCCACATTTTAACGAACTGTGGTGTTACAA & \\
\hline & HPV33_12 & & \\
\hline & $\mathrm{LF}$ & TCTGAGCTGTCACTTAATTGCT & \\
\hline & HPV33_12 & & \\
\hline & LB & ACATTGTAACCTGTTGTCACAC & \\
\hline \multirow[t]{7}{*}{ HPV 39} & HPV39 F3 & TCATCAACCACCAATAAGGAC & L1 \\
\hline & HPV39 B3 & GCCAGTATCAATCATATCACCA & \\
\hline & HPV39 FIP & CCCAATGGCGGGAACACATTTTGTAGGGATAATGTGTCTGTGG & \\
\hline & & GAGCACTGGGGTAAGGGAAAGTTTTTAGTTCCAAAGGAGGACAG & \\
\hline & HPV39 BIP & $\mathrm{T}$ & \\
\hline & HPV39 LF & GCCTATAATGCACAACTGTGTC & \\
\hline & HPV39 LB & GCATGCAAGCCCAATAATGTAT & \\
\hline \multirow[t]{7}{*}{ HPV 45} & HPV45 F3 & CCTGTTGACCTGTTGTGTTA & E7 \\
\hline & HPV45 B3 & TCCGCCATTGTAGATTATTGG & \\
\hline & HPV45 FIP & GGCTGGTAGTTGTGCATGACTTTTTAGCAATTAAGCGAGTCAGAG & \\
\hline & & TGTTGTAAGTGTGACGGCAGAATTTTCTGCTGTAGTGTTCTAAGG & \\
\hline & HPV45 BIP & TC & \\
\hline & HPV45 LF & AACTCCATCTGCTTCATCGTT & \\
\hline & HPV45 LB & AGTAGAGAGCTCGGCAGA & \\
\hline \multirow[t]{6}{*}{ HPV 51} & HPV51 F3 & GCAATGCACAACAAGATGTT & L1 \\
\hline & HPV51 B3 & TGGAATTACCATATGTGTCTGC & \\
\hline & HPV51 FIP & TCTCCTGGAGGTACAGGTGTGTTTTGCTGTGCTCCACCTATTG & \\
\hline & HPV51 BIP & CTCTGTCATTCAGGATGGCGATTTTTCTGATTTGGTGGCCTGTAG & \\
\hline & HPV51 LF & TGCATGTAGTGCCAATACCC & \\
\hline & HPV51 LB & CAGGGTTTGGAGCTATGGATT & \\
\hline \multicolumn{4}{|c|}{ Multiplex 2} \\
\hline \multirow[t]{7}{*}{ HPV 35} & HPV35 F3 & GTGTCAGTGTCTAAGGTTGTTA & L1 \\
\hline & HPV35 B3 & TCTGTACCAGAGTTACCAACA & \\
\hline & & GGTGACCCACAGCTAATAGCCTTTTGCACTGATGAATATGTAACA & \\
\hline & HPV35 FIP & CG & \\
\hline & HPV35 BIP & CTGCCTCCCAGCGTTTAGTTTTTTTACACCTAATGGCTGACCA & \\
\hline & HPV35 LF & AGAACTGCCTGCATGATAGTAG & \\
\hline & HPV35 LB & GTACAGGAGTTGAAGTAGGTCG & \\
\hline HPV 56 & HPV56 F3 & CAAACATTCCCAAAGTTAGTGC & L1 \\
\hline
\end{tabular}




\begin{tabular}{|c|c|c|c|}
\hline & HPV56 B3 & \multicolumn{2}{|l|}{ TTGCTTGCCATCAACTGAT } \\
\hline & HPV56 FIP & \multicolumn{2}{|l|}{ TCCAAACCTACACATGCCCACTTTTTAAGTTTGGGCTTCCAGATAC } \\
\hline & HPV56 BIP & \multicolumn{2}{|l|}{ CGGACAGCCTTTAGGTGCTTTTTAACTTTCAGTATCATCCAGCC } \\
\hline & HPV56 LF & \multicolumn{2}{|l|}{ CTAACCGTTCCTGGTCCG } \\
\hline & HPV56 LB & \multicolumn{2}{|l|}{ GGCTAAGTGGCCATCCATT } \\
\hline \multirow[t]{6}{*}{ HPV 58} & HPV58 F3 & TAGGGTGCGTTTACCTGA & \multirow[t]{6}{*}{ L1 } \\
\hline & HPV58 B3 & GGAGGTTTACAGCCAATTAAAC & \\
\hline & HPV58 FIP & ACCCAATGGCTGTCCCCTATTTTCCTGATACACAACGTTTGGT & \\
\hline & HPV58 BIP & GGCGTAAGTGGTCATCCTTGTTTTTTTATCAGACCCTGGCTGTG & \\
\hline & HPV58 LF & TCAAGGCCTACACATGCC & \\
\hline & HPV58 LB & TGACACTGAAACCAGTAACAGA & \\
\hline \multirow[t]{6}{*}{ HPV 68} & HPV68 F3 & ATTAGGTGTTGGCCTTAGTG & \multirow[t]{6}{*}{ L1 } \\
\hline & HPV68 B3 & TCCATAGCACCATATCCTGT & \\
\hline & HPV68 FIP & CAATGGCAGGAACACAGCCTATTTTATCCTAAGGACAGTAGGGAC & \\
\hline & HPV68 BIP & GAGCACTGGGCCAAAGGTAATTTTCTAATTCCAATGGTGGACAGT & \\
\hline & HPV68 LF & TGCGTTTGTTTATAGTCCACTG & \\
\hline & HPV68 LB & TCTTGTAAGCCTAACAATGTGC & \\
\hline \multicolumn{4}{|c|}{ Multiplex 3} \\
\hline \multirow[t]{7}{*}{ HPV 31} & HPV31 F3 & GACCGTTGTGTCCAGAAG & \multirow[t]{7}{*}{ E7 } \\
\hline & HPV31 B3 & TGTCTATGACATCCTCCTCAT & \\
\hline & & CACTTGGGTTTCAGTACGAGGTTTTTCACAACATAGGAGGAAGGT & \\
\hline & HPV31 FIP & G & \\
\hline & HPV31 BIP & AACATGCGTGGAGAAACACCTTTTTTCATAACAGTGGAGGTCAGT & \\
\hline & HPV31 LF & ATGCTATGCAACGTCCTGT & \\
\hline & HPV31 LB & GTTAGATTTGGAACCTGAGGC & \\
\hline \multirow[t]{8}{*}{ HPV 52} & HPV52 F3 & GGTCTAACTCTGGCAATACTG & \multirow[t]{8}{*}{ L1 } \\
\hline & HPV52 B3 & CCATGACGAAGGTATTCCTTAA & \\
\hline & & TATGCCATTATTGTGGCCCTGCTTTTTATGGGTAACCTCAGAATCCC & \\
\hline & HPV52 FIP & A & \\
\hline & & GGGCAATCAGTTGTTTGTCACATTTTTCCTCAGCACATAAAGTCATG & \\
\hline & HPV52 BIP & $\mathrm{T}$ & \\
\hline & HPV52 LF & CACGTTGTAACCAGTACGGT & \\
\hline & HPV52 LB & TGGATACCACTCGTAGCACTA & \\
\hline \multirow[t]{4}{*}{ HPV 59} & HPV59 F3 & GATGCTTATGGAGACAGTATGT & \multirow[t]{4}{*}{ L1 } \\
\hline & HPV59 B3 & GAATGTATGACATTACCTCCGT & \\
\hline & HPV59 FIP & TGAGCCTTGTGCAGCCAATTTTTACTGACATACGTGCCAAC & \\
\hline & HPV59 BIP & ACTACTCGCAGCACCAATCTTTTTTTTCCACATGTCTGGCATATTC & \\
\hline
\end{tabular}




\begin{tabular}{llll} 
& HPV59 LF & GAATCAGAAGTAACCACAGACC & \\
& HPV59 LB & GTGTGCTTCTACTACTTCTTCT & \\
\hline HPV 66 & HPV66 F3 & GCAATGAGCAATTGGACAG & \\
& HPV66 B3 & ACGTTACTGTTAACGCACC & \\
& AGCTTGTCTAGCTTGCTGTGGTTTTGAGGATGAGGATGAGGATG & \\
& HPV66 FIP & A & \\
& HPV66 BIP & TGTAAGTGTGAGTTGGTGGTGCTTTTTACCACACGTAGCTCCTC & \\
& HPV66 LF & CGCTCCAGCAAATGGTCTA & \\
HPV66 LB & AGTTGGACATTCAGAGTACCAA & \\
\hline
\end{tabular}

Table S2 Cervical swab samples

\begin{tabular}{|c|c|c|c|c|}
\hline Sample ID & $\begin{array}{l}\text { HPV type detected } \\
\text { (Roche linear array) }\end{array}$ & $\begin{array}{l}\text { Sample } \\
\text { collection } \\
\text { date }\end{array}$ & Sample origin & Sample type \\
\hline 11 & 6 & $1999 / 2 / 5$ & Urine & Cell pellet \\
\hline 9 & 16 & $1999 / 2 / 3$ & Urine & Cell pellet \\
\hline 10 & 16 & $1999 / 2 / 5$ & Urine & Cell pellet \\
\hline 21 & 16 & $2002 / 2 / 22$ & Cervix & Swab \\
\hline 22 & 16 & $2002 / 2 / 22$ & Vagina & Self swab \\
\hline 25 & 16 & $2002 / 3 / 25$ & Vagina & Self swab \\
\hline 36 & 16 & $2004 / 10 / 27$ & Cervix & Swab \\
\hline 47 & 16 & $2006 / 10 / 31$ & Cervix & Swab \\
\hline 4 & 18 & $1998 / 11 / 2$ & Urine & Cell pellet \\
\hline 5 & 18 & $1998 / 11 / 17$ & Urine & Cell pellet \\
\hline 26 & 18 & $2002 / 3 / 25$ & Vagina & Self swab \\
\hline 28 & 18 & $2002 / 4 / 15$ & Vagina & Self swab \\
\hline 48 & 18 & $2004 / 6 / 16$ & Cervix & Swab \\
\hline 17 & 31 & $2001 / 5 / 14$ & Vagina & Self swab \\
\hline 46 & 33 & $2000 / 5 / 24$ & Vagina & Self swab \\
\hline 2 & 39 & $1998 / 8 / 4$ & Urine & Cell pellet \\
\hline 37 & 39 & $2004 / 12 / 9$ & Cervix & Swab \\
\hline 6 & 45 & $1998 / 12 / 7$ & Urine & Cell pellet \\
\hline 39 & 45 & $2005 / 1 / 12$ & Cervix & Swab \\
\hline 8 & 51 & $1999 / 2 / 23$ & Urine & Cell pellet \\
\hline 7 & 52 & $1998 / 12 / 17$ & Urine & Cell pellet \\
\hline 20 & 52 & $2002 / 2 / 11$ & Vagina & Self swab \\
\hline 24 & 53 & $2002 / 3 / 22$ & Vagina & Self swab \\
\hline 14 & 56 & $1999 / 2 / 26$ & Urine & Cell pellet \\
\hline 23 & 58 & $2002 / 3 / 11$ & Vagina & Self swab \\
\hline 19 & 66 & $2001 / 10 / 15$ & Vagina & Self swab \\
\hline 1 & 68 & $1998 / 5 / 11$ & Urine & Cell pellet \\
\hline 50 & 68 & $2000 / 7 / 26$ & Cervix & Swab \\
\hline
\end{tabular}




\begin{tabular}{|l|l|l|l|l|}
\hline 40 & 84 & $2006 / 4 / 6$ & Cervix & Swab \\
\hline 12 & 11,33 & $1999 / 2 / 8$ & Urine & Cell pellet \\
\hline 44 & $16,18,31,66$ & $2007 / 8 / 1$ & Cervix & Swab \\
\hline 31 & $16,18,45,51,56$ & $2002 / 8 / 5$ & Vagina & Self swab \\
\hline 45 & $16,52,61,62, c p 6108$ & $2007 / 5 / 3$ & Cervix & Swab \\
\hline 42 & 16, w52,54,58,62,83, & $2006 / 4 / 4$ & Cervix & Swab \\
\hline 18 & 84 & & & \\
\hline 13 & $16,18,45$ & $2001 / 9 / 17$ & Vagina & Self swab \\
\hline 38 & $16,18,6,54,66$ & $1999 / 2 / 25$ & Urine & Cell pellet \\
\hline 3 & 31,33 & $2004 / 12 / 28$ & Cervix & Swab \\
\hline 41 & 31,53 & $1999 / 2 / 23$ & Urine & Cell pellet \\
\hline 27 & $35,51,52$ & $2006 / 8 / 8$ & Cervix & Swab \\
\hline 35 & $35,51,59,84$ & $2002 / 4 / 4$ & Vagina & Self swab \\
\hline 43 & 39,42 & $2002 / 8 / 22$ & Vagina & Self swab \\
\hline 16 & 39,56 & $2006 / 8 / 3$ & Cervix & Swab \\
\hline 29 & 58,53 & $1999 / 2 / 26$ & Urine & Cell pellet \\
\hline 15 & $6,18,40,52, w 55,59$ & $2002 / 7 / 8$ & Vagina & Self swab \\
\hline 30 & - & $1999 / 2 / 26$ & Urine & Cell pellet \\
\hline 32 & - & $2002 / 7 / 25$ & Vagina & Self swab \\
\hline 33 & - & $2002 / 8 / 14$ & Cervix & Swab \\
\hline 34 & - & $2002 / 8 / 14$ & Vagina & Self swab \\
\hline 49 & - & $2002 / 9 / 10$ & Cervix & Swab \\
\hline
\end{tabular}

Table S3 Primer and probe sequences of HPVs 45 and 68 for ddPCR

\begin{tabular}{ll}
\hline Primer/probe name & Sequence \\
\hline HPV45-F_1 & CCAAGGGCACACTTTGTAAAC \\
HPV45-R_1 & CCCATAACCTGTATCCACCATATC \\
HPV68-F_1 & CGTGACAGTCCTGGTAGTTATG \\
HPV68-R_1 & TGCCTTGTGCAGCCAATA \\
HPV45-TaqMan probe_1 & FAM-AAAGGAGGACAGTCACCAGGTTGC-BHQ1 \\
HPV68-TaqMan probe_1 & FAM-CCCTCGCCTAGTGGGTCTATGGTA-BHQ1 \\
\hline
\end{tabular}



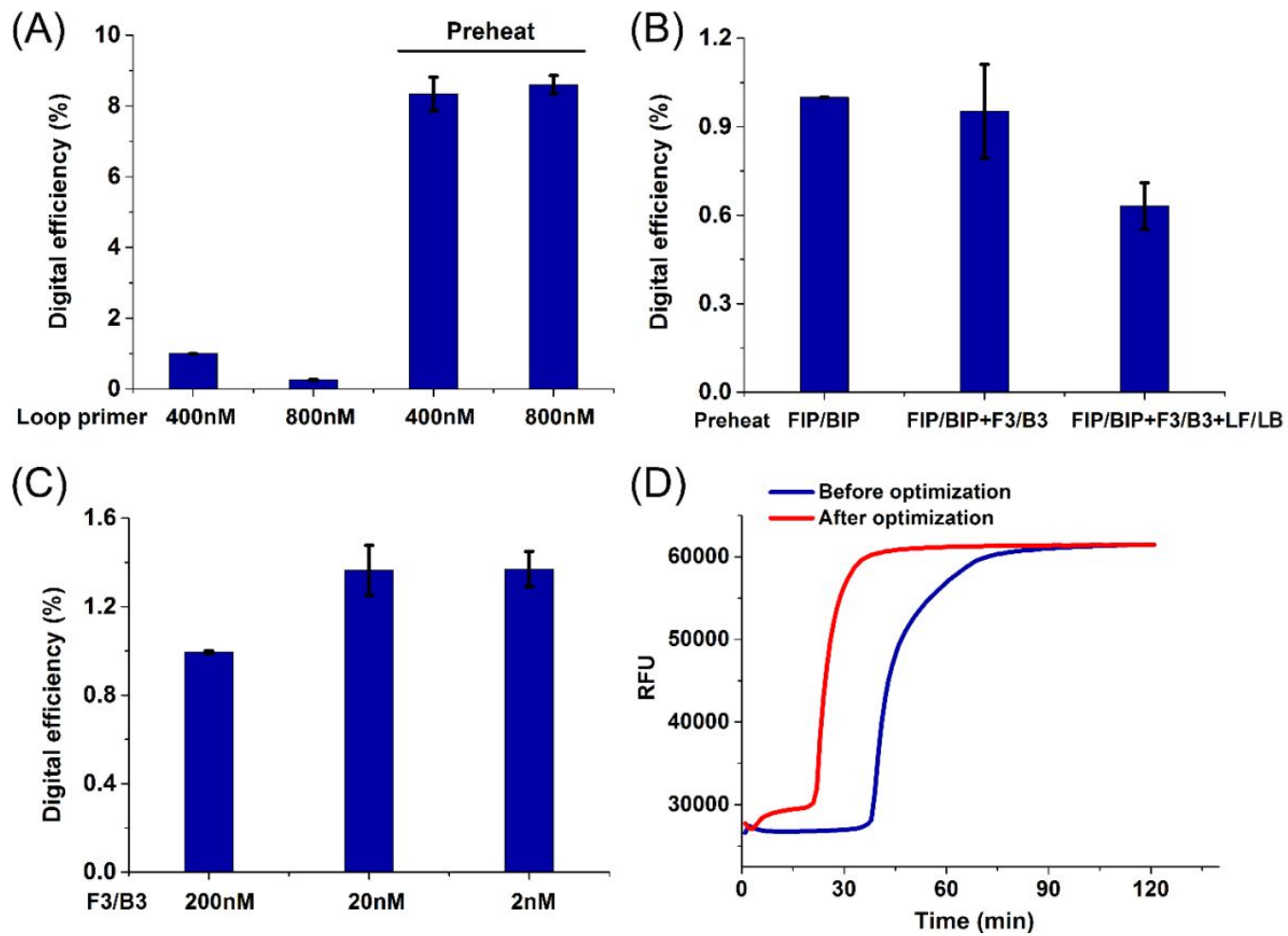

Figure S1 Optimization of dLAMP digital efficiency. (A) Optimization was performed with 1000 copies/ $\mu \mathrm{L}$ HPV 16 and 18 plasmid DNA. The digital efficiency of dLAMP was significantly improved by preheating the HPV DNA with LAMP primers. (B) Normalized digital efficiency of dLAMP when preheating with different combinations of LAMP primers. (C) Normalized digital efficiency of dLAMP with different concentrations of F3/B3 primers. Error bars indicate standard deviation (SD) of technical triplicates. (D) Real-time fluorescence curves of LAMP in tube before (blue) and after (red) optimization. 
(A)

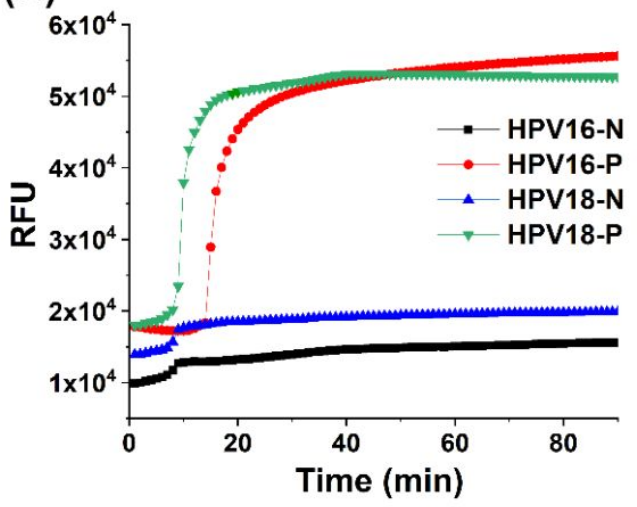

(C)

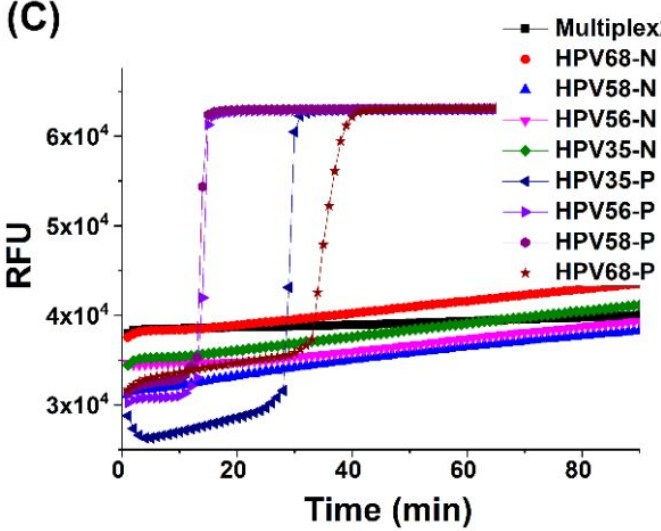

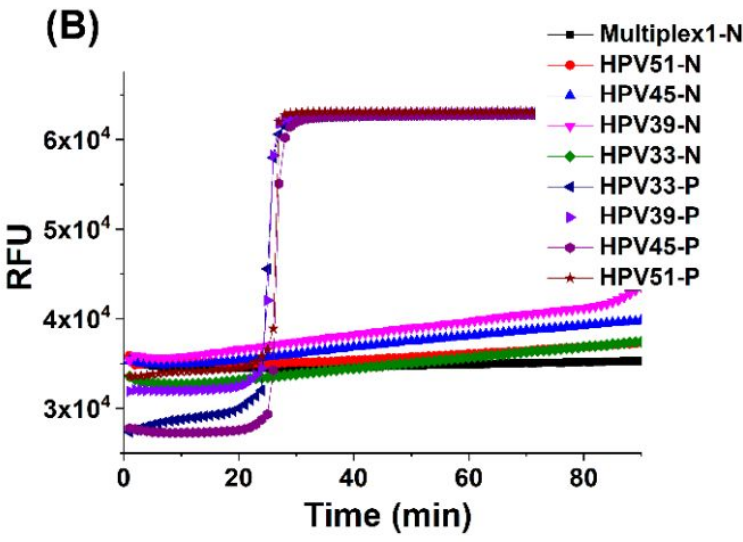

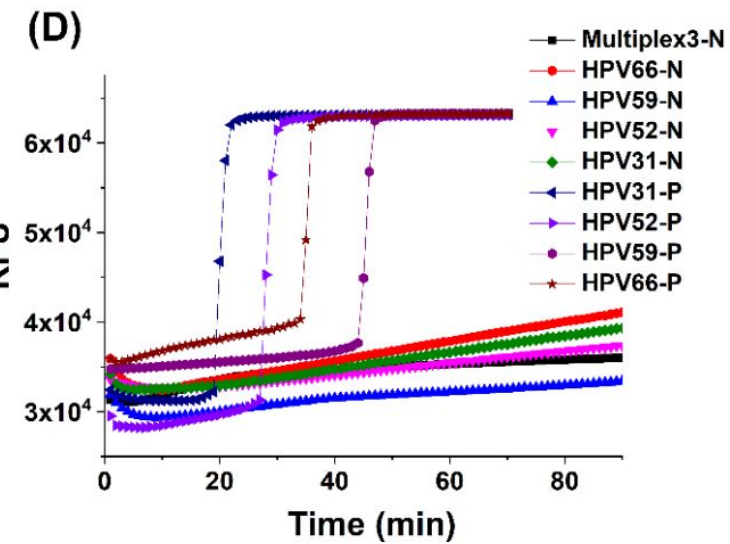

Figure S2 LAMP primer specificity assay. The concentration of each plasmid was $\sim 1.0 \times 10^{5}$ copies/ $\mu$ L. (A) HPV 16 and HPV 18 primer specificities were tested. HPV16-N: HPV 16 primer was used against all plasmids of all 13 other HPV types. HPV16-P: HPV 16 primer was used against all 14 HPV plasmids. HPV 18 primer was tested in the same way. (B) Multiplex 1 primer specificities were tested. The primers were used in a mixture, so we tested the specificities of the primer mix. Multiplex1-N: a primer mix (HPV 33, 39, 45, 51) was used against the other 10 HPV types to verify that the primer in Multiplex 1 did not cross-react with any of the other 10 HPV types. HPV33-N: the primer mix in Multiplex 1 without HPV33 primer (HPV 39, 45, 51) was used against HPV 33 plasmid. HPV33-P: the primer mix (HPV 33, 39, 45, 51) was used against HPV 33 plasmid. We made sure that each primer in Multiplex 1 did not cross-react with any other HPV type. Multiplex 2 (C) and Multiplex 3 (D) primer specificities were tested in the same way. 